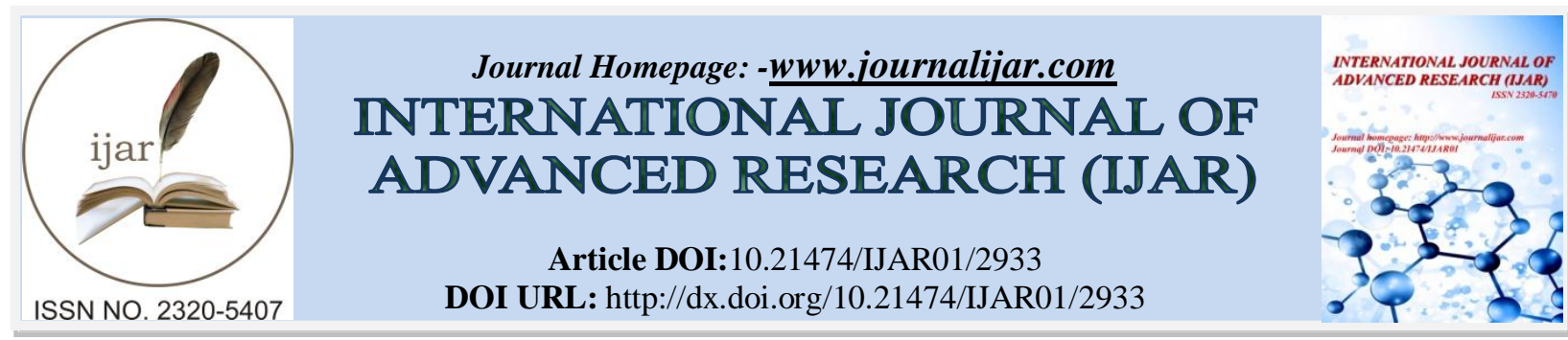

RESEARCH ARTICLE

\title{
SKIN RASH AND DEVELOPMENTAL DELAY IN A NEWBORN WITH PSEUDOHYPOALDESTRONISM.
}

\author{
Olfat Sambas ${ }^{1}$, Yasser Alghanmi ${ }^{1}$, Ammar Yamani ${ }^{2}$ and Ahmad Makeen ${ }^{2}$. \\ 1. Pediatric Endocrinologist, Department of General Pediatric, Hera General Hospital, Makkah, Saudi Arabia. \\ 2. MBBS, Umm Al-Qura University, Makkah, Saudi Arabia.
}

\section{Manuscript Info}

Manuscript History

Received: 25 November 2016

Final Accepted: 27 December 2016

Published: January 2017

\begin{abstract}
Aldosterone is an essential hormone plays an important role in the regulation of plasma electrolytes in the body. In case of Pseudohypoaldestronism, resistance to the action of aldosterone will cause certain electrolytes abnormalities in the form of hyponatremia and hyperkalemia associated with dehydration and metabolic acidosis. Skin and ophthalmological manifestations were reported in rare cases to be part of the type one primary inherited Pseudohypoaldestronism and might consider a possible earlyapproach to differentiate this condition from congenital adrenal hyperplasia in the neonatal period. We report a case of Pseudohypoaldestronism in a newborn with a characteristic dermatological rash accompanied the acute attack with an association of a global developmental delay.
\end{abstract}

Copy Right, IJAR, 2016,. All rights reserved.

\section{Background:-}

Pseudohypoaldestronism (PHA) is a heterogeneous group of disorders that is characterized by electrolyte abnormalities due to renal tubular unresponsiveness or resistance to the action of aldosterone (1). Aldosterone is the major mineralocorticoid secreted by the adrenal gland (2). Nearly 90 percent of the mineralocorticoid activity is carried out by the aldosterone(2). It works on the renal tubules - mainly the principal cells of the collecting tubules and to a lesser extent on the distal tubule and collecting duct resulting in an increaseof reabsorption of sodium and excretion of potassium (2). PHA type one is a rare inherited disease which can be either autosomal recessive or dominant. (3). Clinically, these patients present with renal salt wasting, hypovolemia, hypercholeremicacidosis and hyperkalemia despite the elevated level of aldosterone in plasma and urine (3). The autosomal dominant form is milder than the autosomal recessive (3).In the dominant type, there is aldosterone receptor resistance limited to the kidneys mainly (4).

The autosomal recessive type is considered a systemic disease (4). It involves the sweat glands, lungs and reproductive organs in addition to the kidney (4).PHA type 2 also known as Gordon Syndrome is characterized by hyperkalemic acidosis, hypertension and suppression of renin and aldosterone(3).

PHA symptoms are well known worldwide but the special feature in our case is that we found a generalized maculopapular rash in the skin that presents most commonly on the face and on the trunk. Four cases with a similar presentation to our patient have been reported and the cases were as follow. The first case was reported in 2007in France, the child presented in the first days of life with a rash(5).the second case was in 2014 in Al-Kuwait, the child started to develop Miliariarubra-like eruption on the $4^{\text {th }}$ day of life(6). The same mother of the Kuwaiti child, 13 
months later, delivered a female baby who developed similar manifestations to her brother including the same characteristic rash over the face and trunk on day 2 of life(6). The third case was in 2015 in Turkey of a female baby, on the 7th day of life, she presented with a skin rash noted on her face, her arms and her chest(4). All these cases came with rash in addition to the other symptoms of PHA.

\section{Case History:-}

Our patient is an 11-month-old Syrian boy delivered as a spontaneous vaginal delivery, full term, with uneventful antenatal period. Parents are second-degree cousins. His weight at birth was $3200 \mathrm{~g}$, height $54 \mathrm{~cm}$ and head circumference $36 \mathrm{~cm}$. He was stable, and after the initial screening tests, he was discharged with his mother with no specific complaint. Just 4 days later, he was admitted to the neonatal intensive care unit (NICU)withcomplaints of poor oral intake, excessive crying and multiple episodes of diarrhea.The patient was severely dehydrated, lethargic, cyanosed and afebrile. There was an obvious maculopapular skin rash covering all the body. He was then intubated and connected to a mechanical ventilator for 6 days andkept NPO for 8 days. He wason intravenous fluid and on total parenteral nutrition(TPN). Full septic workup was ordered and antibiotics started. Results of blood chemistry showed hyponatremia NA= $119.9 \mathrm{mEq} / \mathrm{L}$ (135-145), hyperkalemiaK=9.44 mEq/L (3.5-5) and hypercalcemia $\mathrm{Ca}=12.6 \mathrm{mg} / \mathrm{dL}$ (8.5-10.5). Patient received insulin with $10 \%$ dextrose, Ventolin, Ca gluconate and Na resonium for Hyperkalemia. These laboratory results raised the suspicion of congenital adrenal hyperplasia. Hydrocortisone and fludrocortisones were started. 17-hydroxy progesterone, plasma renin and aldosterone levels were sent.Vancomycin was added because gram positive cocciwere found on the blood culture. Moreover, candida was also discovered and Amphotericin B was added. He became stable and he showed improvement in his blood chemistry results.

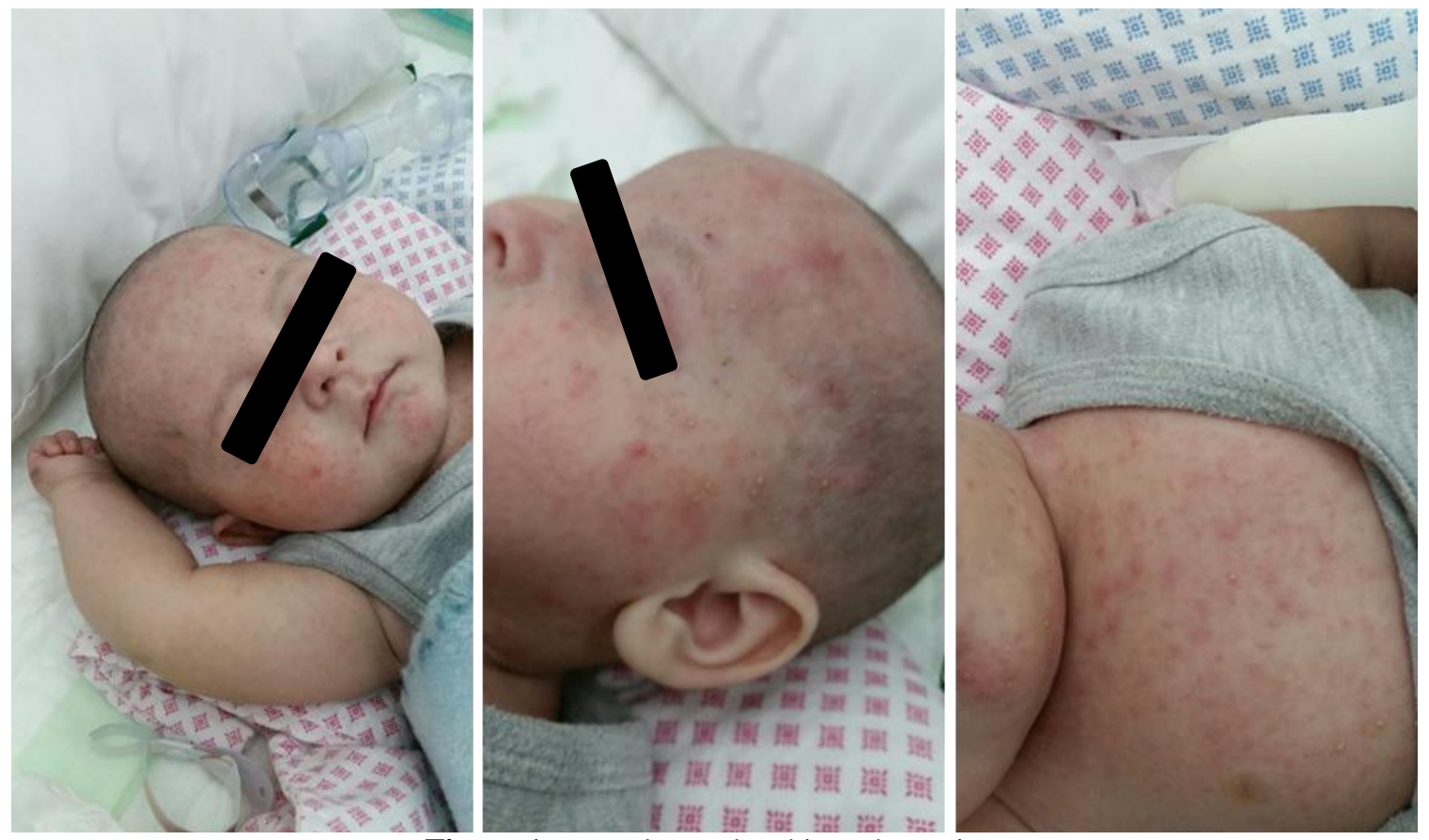

Figure 1:- maculopapular skin rash at crises

On day 11 after admission he again developed hyponatremia $\mathrm{Na}=128 \mathrm{mEq} / \mathrm{L}$ along with hyperkalemiaK=9.22 $\mathrm{mEq} / \mathrm{L}$. Although 17-hydroxy progesterone hormone assay was elevated $=16.49 \mathrm{ng} / \mathrm{ml}(1.70-4.00 \mathrm{ng} / \mathrm{ml})$, howeverboth plasma renin $=500 \mathrm{uIU} / \mathrm{ml}(4.4-46)$ and aldosterone100ng/dL (2.2-35.3) levels were elevated. Abdominal Ultrasound show normal kidneys. These results raised the suspicionof diagnosis of PHAespecially when our patient did not improve with both hydrocortisone and fludrocortisones. $\mathrm{NaCl} 3 \%$ was given orally in addition to Na polystyrene. He stayed in the NICU for approximately 3 months then discharged. Since that time, he has been admitted to our hospital several times with similar symptoms of repeated vomiting, poor oral intake, fever, severe dehydration, hyponatremia and hyperkalemia along with generalized maculopapular skin rash that resolves after each attack. In addition, despite our patient is an 11 month-old, he has obvious developmental delay. He cannot sit without support. Also he uses mama and dada indiscriminately and the primitive reflexes are still present. There is a family history of death of two siblings(boy and girl) at 5 and 9 days of life with similar symptoms but no diagnosis 
was made at that time.He has other 4 living siblings with a good health status and without similar disease. This patient was noted to have frequent admissions (twice per week), but now when he has become a one year old, the admissions rate become less frequent once per month hoping that it will be more spaced in future.
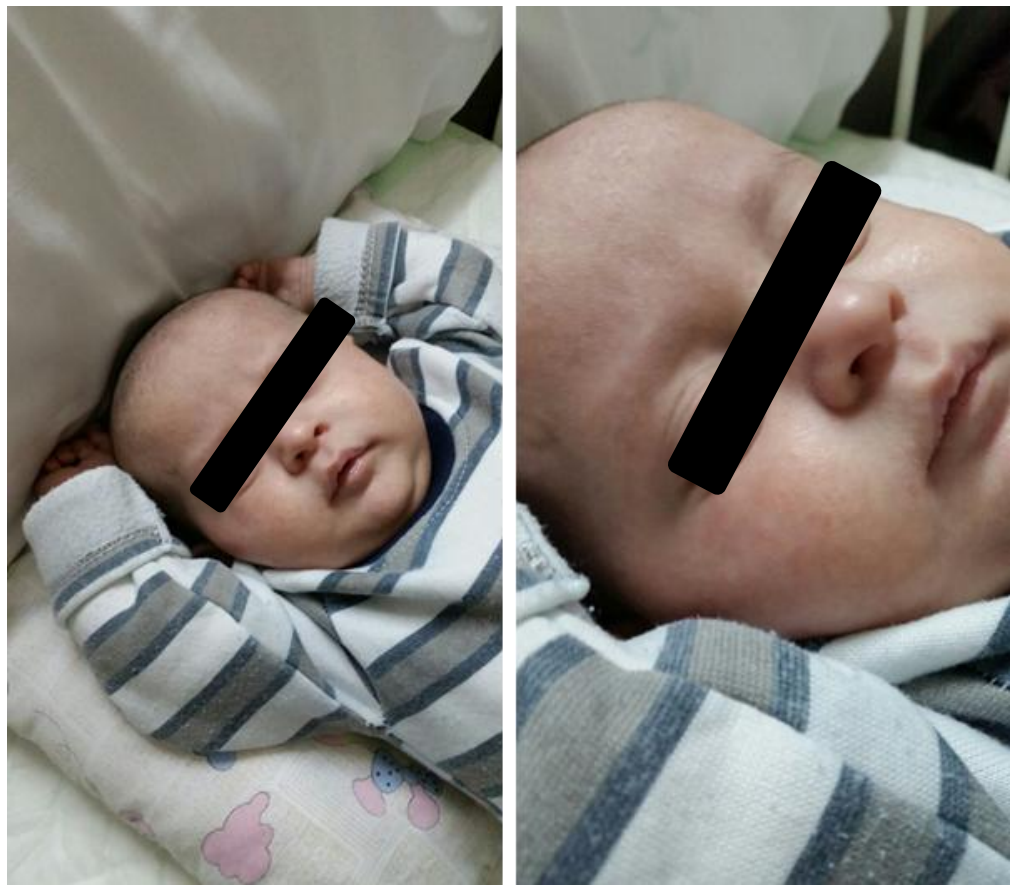

Figure 2:- maculopapular skin rash after resolving

\section{Discussion:-}

Usually in infants who come in their early days of life with symptoms of shock and dehydrationassociated with electrolyte disturbances of hyperkalemia and hyponatremia, congenital adrenal hyperplasia (CAH) must be ruled out. In our case, the diagnosis of $\mathrm{CAH}$ was suspected, therefore treatment with hydrocortisone and fludrocortisone werestarted. Although our patient showed some improvement especially with the correction of hyponatremia and hyperkalemia, however he presented again with similar symptoms a few days later.As a result, further investigations for the cause of these symptoms were indicated and hormonal analysis was sent. Renin and aldosterone levels were elevated and the diagnosis of PHA was suspected despite high level of 17-hydroxy progesterone hormone. Renal ultrasound was done to exclude secondary causes. NACL 3\% and NA polystyrene were started with I.V fluid to correct electrolyte abnormalities and the patient started to improve dramatically.

Maculopapular rash which involves mainly the face and trunk is a characteristic feature that accompanied the acute attacks of the disease (sometimes upper and lower limbs were also involved). This rash is similar to that found in the literature and is a hallmark of the systemic type of PHA. It is not itchy, always disappears with management of acute attack and never leaves any pigmentation or scars. The rash does not respond to local steroid or lotion creams. It may be attributedto the increase concentration of sodium in the sweat which in turn leads to blockage and inflammation of exocrine sweat glands (4-6). We thought that there may be an underlying immune process that may explained by the presence of the rash with each acute attack, high white blood cells count and rapid disappear of rash after use of antibiotics and electrolytes correction. Fever is not always present.

No evidence of eye manifestations were noticed during the assessment of the child (no conjunctivitis, sclerosis, or eye discharge). However, the developmental review revealed a delay in the gross motor function (cannot sit without support), speech (sayDada, Mama indiscriminately) and the presence of primitive reflexesthat are not consistent with his age. This might be due to a cerebral injury earlier in life and an underlying immune-compromised state.

The family history showed two previous deaths of two siblings of a different gender at 5 and 9 days of life who presented with similar symptoms but unfortunately resuscitation failed and no diagnosis was made at that time. The presence of four other living siblings with a good health support the diagnosis of the autosomal recessive systemic PHA. 


\section{Conclusion:-}

Biochemical feature of congenital adrenal hyperplasia and Pseudohypoaldestronism may be indistinguishable initially, especially with a delay in obtaining hormonal assay results. The skin manifestation may give an important diagnostic guideline In which this will influence the management and prevention of recurrence electrolytes disturbance. PHA type one can lead to salt losing crisis and life-threatening hyperkalemia. High dose of sodium supplementation and potassium binding resins are the standard treatment in such case.

\section{Acknowledgment:-}

We thank parents who agreed to publish this case for educational purposes.

\section{References:-}

1. Pseudohypoaldosteronism: Background, Pathophysiology, Etiology [Internet]. Emedicine.medscape.com. 2016 [cited 17 September 2016]. Available from: http://emedicine.medscape.com/article/924100-overview

2. Guyton AHall J. Textbook of medical physiology. Philadelphia: Elsevier Saunders; 2006.

3. Elzouki A. Textbook of clinical pediatrics. [Berlin]: Springer; 2012.

4. Korkut S, Gokalp E, Ozdemir A, Kurtoglu S, Demirtas S, Gul U, et al. Dermal and Ophthalmic Findings in Pseudohypoaldosteronism. Journal of clinical research in pediatric endocrinology. 2015 Jun;7(2):155-8. PubMed PMID: 26316441. Pubmed Central PMCID: 4563190.

5. Argoubi H, Fitchner C, Richard O, Lavocat MP, Cambazard F, Stephan JL. [Pustular miliaria rubra and systemic type $1 \mathrm{~b}$ pseudohypoaldosteronism in a newborn]. Annales de dermatologie et de venereologie. 2007 Mar;134(3 Pt 1):253-6. PubMed PMID: 17389851. Miliaire rouge et pseudo hypoaldosteronemisme de type 1b de revelation neonatale.

6. Eliwa MS, El-Emmawie AH, Saeed MA. Ocular and skin manifestations in systemic pseudohypoaldosteronism. BMJ case reports. 2014;2014. PubMed PMID: 24654255. Pubmed Central PMCID: 3962973. 